\title{
Brain Tumor Classification with Hybrid Algorithm Convolutional Neural Network-Extreme Learning Machine
}

\author{
Radical Rakhman Wahid ${ }^{1}$ \\ Informatics Department \\ UPN "Veteran" Jawa Timur \\ Surabaya, Indonesia \\ 17081010068@student.upnjatim.ac.id
}

\author{
Fetty Tri Anggraeni ${ }^{2}$ \\ Informatics Department \\ UPN "Veteran" Jawa Timur \\ Surabaya, Indonesia \\ fettyanggraeny.if@upnjatim.ac.id
}

\author{
Budi Nugroho ${ }^{3}$ \\ Informatics Department \\ UPN "Veteran" Jawa Timur \\ Surabaya, Indonesia \\ budinugroho.if@upnjatim.ac.id
}

\begin{abstract}
Brain tumor is a disease that attacks the brains of living things in which brain cells grow abnormally in the area around the brain. Various ways have been done to detect this disease, one of which is through the anatomical approach to medical images. In this study, the authors propose a Convolutional Neural Network (CNN)-Extreme Learning Machine (ELM) hybrid algorithm through Magnetic Resonance Imaging (MRI). ELM was chosen because of its superiority in the training process, which is faster than iterative machine learning algorithms, while $\mathrm{CNN}$ was chosen to replace the traditional feature extraction process. The result is CNN-ELM, which has 8 filters in the convolution layer and 6000 nodes in the hidden layer, has the best performance compared to CNN-ELM another model which has different number of filters and number of nodes in the hidden layer. This is evidenced by the average value of precision, recall, and F1-score which is 0.915 while the accuracy of the test is $91.4 \%$.
\end{abstract}

Keywords-Classification; Hybrid Algorithm; Digital Image Processing; Brain Tumor; Neural Network

\section{INTRODUCTION}

Brain tumor is a disease that attacks the human brain in which brain cells grow abnormally in the brain. The growth is caused by DNA mutations in cells. Cells that should grow and die at one time actually live and multiply uncontrollably until they become tumors [1]. Various methods are used to detect the presence of brain tumors early, one of which is through an anatomical approach to medical images [2]. Some examples of medical images are X-rays, CT-scans, and MRI. The image quality on X-rays is less good, so a lot of information is missing (lack of detail), the use of CT-scans is more suitable for viewing changes in bone structure. CT-scans, if used in cases of brain tumor detection, is still not optimal because brain tumors contain soft tissues. MRIs are very sensitive and successfully provide good image information [3], so the MRI is able to provide a clear picture between soft tissues and hard tissues in the brain.

Neural network is a machine learning algorithm that can be customized flexibly. Because of its flexibly customizable nature, it is not impossible if one neural network structure can be combined with another neural network structure so a new artificial neural network structure (hybrid model) can be formed. This combination is able to cover the shortcomings of each algorithm and improve its performance both in terms of accuracy and speed of classification [4]. To handle images data, a neural network algorithm that shows significant performance is the Convolutional Neural Network (CNN) [5]. On the other hand, Extreme Learning Machine (ELM) is a neural network whose training process does not require backpropagation so that the training process can be faster because it does not need to update the weights as well [6]. The two algorithms can be combined into a hybrid algorithm as well [7].

The contribution of this work is to get the advantage of CNN-ELM hybrid model in MRI classification. So, the convolution and pooling layers on CNN can be used as feature extractors while ELM is used to train weights without backpropagation and perform the classification. As well, providing novel insights about the CNN-ELM models' performance evaluation. To the extent of doing a literature search, the literature that specifically discussed CNN-ELM aftermath for medical imaging is only a few.

\section{PREVIOUS RESEARCH}

Back in 2015, Yu et al. [7] used a hybrid CNN-ELM algorithm to classify wireless capsule endoscopy images. Where CNN used to be a feature extractor and ELM for the classifier. In the end, their hybrid model can achieve the accuracy by $97.25 \%$. Study by Pashaei, et al. [8] also used a hybrid CNN-ELM algorithm to classify brain tumors from MRI data. They also used CNN as a feature extractor and ELM as a classifier. Their CNN-ELM can achieve classification accuracy by $93.68 \%$ as well. The use of the hybrid CNN-ELM algorithm can also be found in non-medical image-based research, like Peng. et al [9], Kannojia \& Jaiswal [10], Guo \& Ding 2015 [11], Mingxing et al. [12], Sharma et al. [13], and Zhou \& Tan [14].

We found two studies that use the same dataset as us. First, study by Shabrina, Arief, \& Fuady [15], they use Support 
Vector Machine to classify (C-SVM) the brain tumor and get $76 \%$ accuracy on test data. To improve the performance, Rakhman, Tri, \& Nugroho [16] use ELM. They could get the recall, precision, \& F1 score by 0.86 and accuracy score achieved $86 \%$. What a good improvement by number.

\section{RESEARCH METHODS}

The process starts from the data preprocessing, followed by building a hybrid CNN-ELM model, then train the model on preprocessed data after that the hybrid model that has passed the performance training is test on the test data. In the end, the performance of the algorithm can be known when it comes to the model evaluation. The same process from model building to model evaluation will be repeated depend on how many parameters combinations that want to try it out.

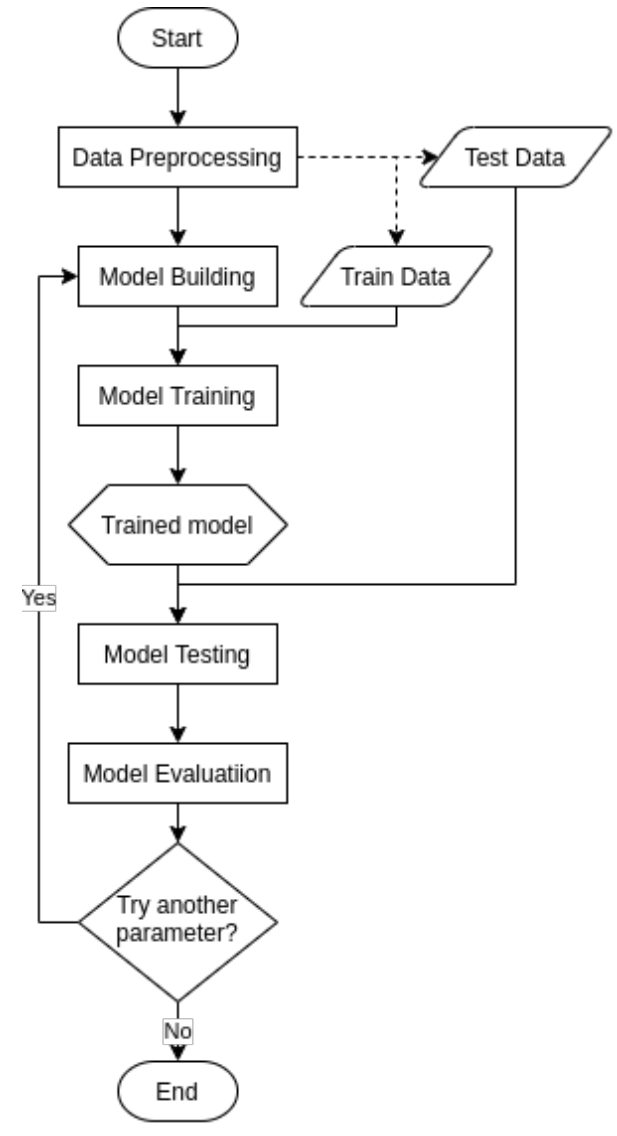

Fig. 1. The experiment flowchart

The hardware specifications used by the authors are Intel ${ }^{\mathrm{R}}$ Core $^{\mathrm{TM}}$ i5-7200U $2.5-3.1 \mathrm{GHz}$ as a processor and DDR4 12 GB RAM. Hybrid model trained on a 4 cores CPU in Ubuntu 20.04 OS. CNN-ELM builds on top of NumPy [17] and ScikitImage [18] to process digital image data.

\section{A. Dataset}

We use Brain MRI Images for Tumor Detection Dataset [19] in this study, which is the data set is open to the public so it can be used by everyone. The dataset contains 253 MRI images which look like grayscale images (1 channel), but there are some images which are not grayscale as well (RGB, 3 channel). From Kaggle, Navoneel Chakrabarty [19] uploaded the Brain MRI Images for Tumor Detection Dataset, he is someone who creates and arranges the dataset. From 253 images it is divided by two main directories. The first directory is Tumor and No Tumor. No Tumor directory has 98 images while Tumor directory has 155 images. These are the image samples from the dataset as well:
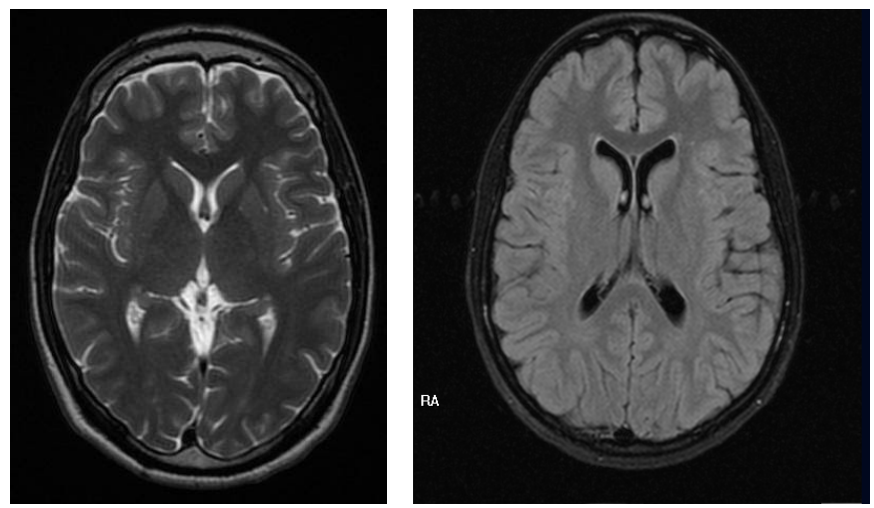

Fig. 2. Image samples of No Tumor Category

Figure 2 are two samples of the No Tumor category. At least the images in that category have a total percentage of $38.7 \%$ of the overall dataset.
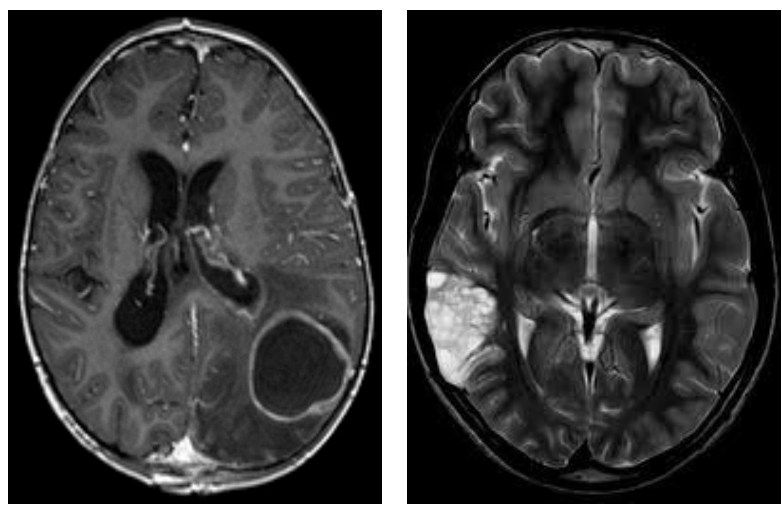

Fig. 3. Image samples of Tumor Category

Figure 3 are also two samples of the Tumor category. At least the images in that category have a total percentage of $61.3 \%$ of the overall dataset as well.

\section{B. Image Preprocessing}

First, we transform the image's color by changing some of the images that have 3 channels (RGB) into grayscale (1 channel). After each image has the same amount of channel, then change the images resolution from its original size to $50 \times 50$ pixels. Considering the data distribution is imbalanced, we apply the oversampling technique to balancing the distribution [20]. For the oversampling phase we rotated some of the images, which were categorized as No Tumor by $180^{\circ}$ so from the initial number of images from 98 increased to 155, the same number like the images from Tumor category so the total data after oversampling is, 310 in number. After that, the value of each element in the matrix normalized by changing the range using the Min-Max normalization. Where the initial range is $0-255$, we change it to $0-1$ to make the distribution of 
values more even [21]. The last step is, divide the dataset into two parts, first train data and test data. Where the train data consisting of 217 images (70\% dataset) are used to train the CNN-ELM hybrid model while test data consisting of 93 images (30\% dataset) are used to test the performance of the model as well.

\section{Hybrid CNN-ELM Algorithm}

The illustration of CNN-ELM hybrid algorithm shown in Figure 4 below. There are two main architectures: CNN and ELM, where CNN used to be a feature extractor and ELM as a classifier. The CNN architecture proposed only consists of one convolutional layer and one pooling layer. For the ELM we just use one hidden layer only, between input and output layer as well.

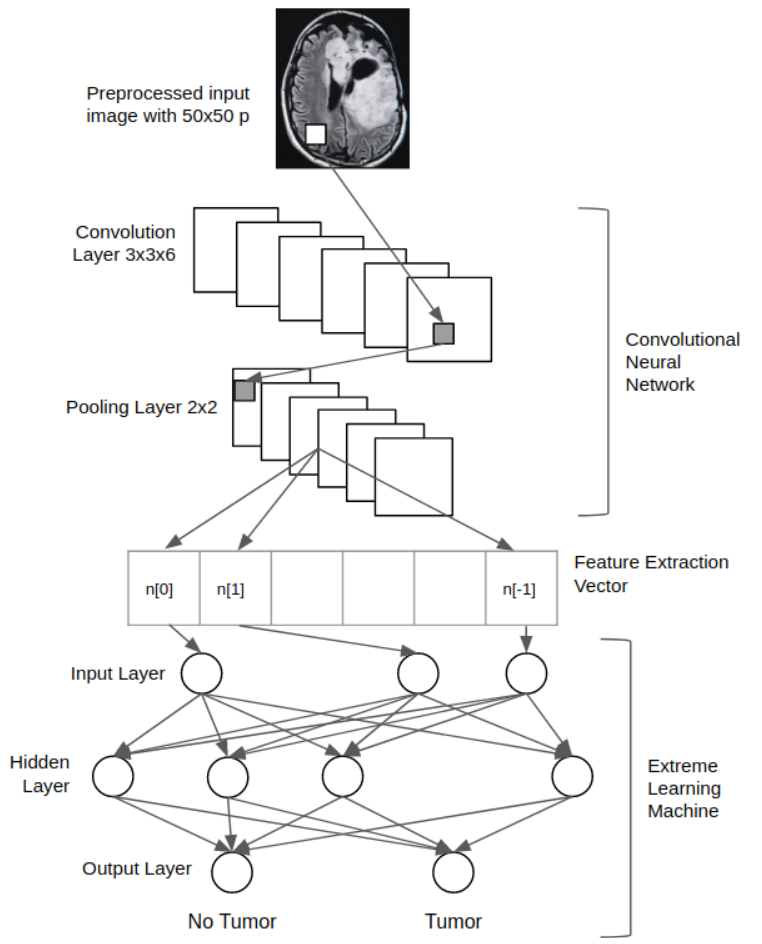

Fig. 4. The illustration of CNN-ELM architecture

The main flow of CNN-ELM starts from the image which enters the convolutional layer, then the image matrix activated by ReLU before entering the (max) pooling layer as well. Afterward each processed image matrix turned into a onedimensional vector to enter the input layer of ELM. The flattened image data, then calculated by general calculation in the neural network before entering the ELM hidden layer and activated by Sigmoid function. After we have activated values, the process continues to the calculation from hidden layer to output layer of ELM by using the formula below in order to get a classification result, as well:

$$
W_{h-o}=\left(X^{T} X\right)^{-1} X^{T} y
$$

\section{Model Training and Model Testing}

The training was carried out on 217 MRI preprocessed images in the train data as well. It should be noted that the sequence of images, kernel values in the convolution layer, weights and bias values in ELM at each start of a model training is always initialized randomly. In order to get the best metric this study carried out with nine different scenarios adjusted for the choice of the number of filters in the convolution layer $(\mathrm{CNN})$ and the number of nodes in the hidden layer (ELM). The variations in the number of nodes in the hidden layer are 4000, 5000, and 6000 nodes, while variations of filters in the convolution layer are 8,6 , and 10 . It should be noted that the more nodes in the hidden layer and the number of filters at the convolution layer, the more memory (RAM) the computer must allocate [22].

The testing process also performed in preprocessed 93 images which in the test data on each different scenario. After the testing phase, the prediction value will be compared with the original label value contained in the test data, to calculate the number of correct predictions. After that the confusion matrix can be used to calculate the precision, recall, accuracy, and F1 score [23].

\section{RESUlTS AND DiscUSSIONS}

TABLE I. THE EVALUATION RESULTS OF CNN-ELM WITH 6 CONVOLUTIO FILTER

\begin{tabular}{|c|c|c|c|c|c|}
\hline Nodes & Classes & Precision & Recall & F1 & Accuracy (\%) \\
\hline \multirow{2}{*}{4000} & Tumor & 0.82 & 0.7 & 0.76 & \multirow{2}{*}{78.5} \\
\cline { 2 - 5 } & No Tumor & 0.76 & 0.86 & 0.81 & \\
\hline \multirow{2}{*}{5000} & Tumor & 0.74 & 0.67 & 0.7 & \multirow{2}{*}{72} \\
\cline { 2 - 5 } & No Tumor & 0.71 & 0.77 & 0.73 & \\
\hline \multirow{2}{*}{6000} & Tumor & 0.74 & 0.62 & 0.67 & \multirow{2}{*}{69.9} \\
\cline { 2 - 5 } & No Tumor & 0.67 & 0.78 & 0.72 & \multirow{2}{*}{} \\
\hline
\end{tabular}

In the experiment with 4000 nodes in the hidden layer it obtained varying precision, recall, and F1 score from the range 0,7 to 0,86 with the accuracy $78,5 \%$. For 5000 nodes in the hidden layer also have varying precision, recall, \& F1 score from 0,7 to 0,77 with $72 \%$ accuracy. CNN-ELM with 6000 nodes in hidden layer achieve $69,9 \%$ accuracy also with a variety of precision, recall, and F1 score in two categories. The best CNN-ELM learning model with 6 filters is on a network with 4000 nodes.

TABLE II. THE EVALUATION RESULTS OF CNN-ELM WITH 8 CONVOLUTIO FILTER

\begin{tabular}{|c|c|c|c|c|c|}
\hline Nodes & Classes & Precision & Recall & F1 & Accuracy (\%) \\
\hline \multirow{2}{*}{4000} & Tumor & 0.87 & 0.9 & 0.89 & \multirow{2}{*}{87} \\
\cline { 2 - 5 } & No Tumor & 0.87 & 0.83 & 0.85 & \\
\hline \multirow{2}{*}{5000} & Tumor & 0.8 & 0.73 & 0.77 & \multirow{2}{*}{76.3} \\
\cline { 2 - 5 } & No Tumor & 0.73 & 0.8 & 0.76 & \\
\hline \multirow{2}{*}{6000} & Tumor & 0.92 & 0.92 & 0.92 & \multirow{2}{*}{91.4} \\
\cline { 2 - 5 } & No Tumor & 0.91 & 0.91 & 0.91 & \\
\hline
\end{tabular}


In the experiment with 4000 nodes in the hidden layer obtains precision, recall, and F1 score greater than equal to 0.87 with the accuracy $87 \%$. For 5000 nodes in the hidden layer have varying precision, recall, \& F1 score from 0,73 to 0,8 with $76.3 \%$ accuracy. CNN-ELM with 6000 nodes in hidden layer achieve $91.4 \%$ accuracy with an average of precision, recall, and F1 score 0.915 . Can be concluded that the CNN-ELM learning model with 8 filters on a network with 6000 nodes is better than the other.

TABLE III. THE EVALUATION RESULTS OF CNN-ELM WITH 10 CONVOLUTIO FILTER

\begin{tabular}{|c|c|c|c|c|c|}
\hline Nodes & Classes & Precision & Recall & F1 & Accuracy (\%) \\
\hline \multirow{2}{*}{4000} & Tumor & 0.91 & 0.83 & 0.87 & \multirow{2}{*}{87} \\
\cline { 2 - 5 } & No Tumor & 0.84 & 0.91 & 0.87 & \\
\hline \multirow{2}{*}{5000} & Tumor & 0.84 & 0.7 & 0.76 & \multirow{2}{*}{75} \\
\cline { 2 - 5 } & No Tumor & 0.67 & 0.82 & 0.74 & \\
\hline \multirow{2}{*}{6000} & Tumor & 0.82 & 0.77 & 0.8 & \multirow{2}{*}{81.7} \\
\cline { 2 - 5 } & No Tumor & 0.81 & 0.86 & 0.83 & \multirow{2}{*}{} \\
\hline
\end{tabular}

In the experiment with 4000 nodes in the hidden layer it obtained varying precision, recall, and $\mathrm{F} 1$ score from the range 0,83 to 0,91 with the accuracy $86 \%$. For 5000 nodes in the hidden layer also have varying precision, recall, \& F1 score from 0,7 to 0,84 with $75 \%$ accuracy. CNN-ELM with 6000 nodes in hidden layer achieve $81.7 \%$ accuracy also with a variety of precision, recall, and F1 score in two categories. The best CNN-ELM learning model with 10 filters is on a network with 4000 nodes.

TABLE IV. COMPARISON OF EVALUATION METRICS FROM A DIFFERENT MACHINE LEARNING ALGORITHMS.

\begin{tabular}{|c|c|c|c|c|}
\hline Architecture & Precision & Recall & F1 & $\begin{array}{c}\text { Accuracy } \\
\text { (\%) }\end{array}$ \\
\hline C-SVM & 0.83 & 0.75 & 0.788 & 76 \\
\hline ELM & 0.86 & 0.86 & 0.86 & 86 \\
\hline CNN-ELM & 0.915 & 0.915 & 0.915 & 91.4 \\
\hline
\end{tabular}

C-SVM algorithm have the precision score 0.83 , recall 0.75, F1 0.788 and accuracy $76 \%$ [15]. The evaluation result then improved with a different machine learning algorithm, it is ELM, in a different study. By using ELM, the average score of precision, recall, \& F1 is 0,86 and test accuracy $86 \%$ [16]. In this study, with a variety of experiments our CNN-ELM can get the average score of precision, recall, \& F1 0,915 with $91,4 \%$ accuracy as well.

\section{CONCLUSiOnS}

There are several image preprocessing techniques which can affect the final result, such as changing the image size, converting the image to grayscale, oversampling data, normalizing values, etc. The performance of CNN-ELM can be optimized by setting the size of the kernel in the convolution layer, submatrix size/dimension of the pooling layer, the number of nodes in the hidden layer and choosing the right activation function for the hidden and output layer, etc. Nevertheless, CNN-ELM with 8 filters and 6000 nodes in the hidden layer gained the best result. It is able to get an average precision, F1-score, and recall of 0.915 and $91.4 \%$ accuracy. The improvements that can be made in the near future are that the data used should be more varied, and image segmentation phase can be added. A trained learning model can be embedded in an online diagnosis system application so that it does not stop at the research phase as well.

\section{REFERENCES}

[1] Fadila, I., Nov. (2020). Tumor Otak : Gejala, Penyebab, dan Pengobatan, Available: https:/hellosehat.com/kanker/kankerotak/tumor-otak/.

[2] Suta, I., Hartati, R., dan Divayana, Y., Juni. 2019. "Diagnosa Tumor Otak Berdasarkan Citra MRI (Magnetic Resonance Imaging)". Majalah Ilmiah Teknologi Elektro Universitas Udayana 18, 2:149--153.

[3] Riley, R., Murphy, J., dan Higgins, T., Jan. 2018. "MRI imaging in pediatric appendicitis,". Journal of Pediatric Surgery Case Reports 31:88-89.

[4] Psichogios, D., dan Ungar, L., 1992. "A hybrid neural network-first principles approach to process modeling". AIChE Journal 38, 10:14991511

[5] Simonyan, Karen, dan Andrew Z., 2014 "Very deep convolutional networks for large scale image recognition." arXiv preprint 1409.1556.

[6] Guang-Bin, H., Qin-Yu Z., dan Chee-Kheong, S., 2006. "Extreme learning machine: Theory and applications". Neurocomputing 70, 1:489 -501 .

[7] Yu, J., Chen, J., Xiang, Q., dan Zou, Y., 2015. "A hybrid convolutional neural networks with extreme learning machine for WCE image classification". IEEE International Conference on Robotics and Biomimetics (ROBIO). 1822-1827

[8] Pashaei, A., Sajedi, H., dan Jazayeri, N., 2018. "Brain Tumor Classification via Convolutional Neural Network and Extreme Learning Machines". $20188^{\text {th }}$ International Conference on Computer and Knowledge Engineering (ICCKE).A

[9] Peng, W., Xiaomin, Z., dan Yan, H., 2019. "A Method Combining CNN and ELM for Feature Extraction and Classification of SAR Image." Journal of Sensors Hindawi 2019, 6134610.A

[10] Kannojia, S., dan Jaiswal, G., 2018. "Ensemble of Hybrid CNN-ELM Model for Image Classification". 2018 5 International Conference on Signal Processing and Integrated Networks (SPIN).

[11] Guo, L., dan Ding, S., 2015. "A hybrid deep learning CNN-ELM model and its application in handwritten numeral recognition". Journal of Computational Information Systems 11.

[12] Mingxing, D., Kenli, L., Canqun, Y., Keqin, L., 2018 "A Hybrid Deep Learning CNN-ELM for Age and Gender Classification". Neurocomputing 275. 448-461.

[13] Sharma, J., Ole-Christoffer, G., dan Morten, G., 2018. "Deep CNNELM Hybrid Models for Fire Detection in Images”. 27 "International Conference on Artificial Neural Networks III.

[14] Zhou, S., dan Tan, B., 2019. "Electrocardiogram soft computing using hybrid deep learning CNN-ELM". Applied Soft Computing 86.

[15] Shabrina, A., Arief, T., Fuady, A., 2020. "Klasifikasi Tumor Otak pada Citra Magnetic Resonance Image dengan Menggunakan Metode Support Vector Machine”. Jurnal Teknik ITS 9. 1:2301-9271

[16] Rakhman, R., Tri, F., dan Nugroho, B., 2020. "Implementasi Metode Extreme Learning Machine untuk Klasifikasi Tumor Otak pada Citra Magnetic Resonance Imaging". Seminar Nasional Informatika Bela Negara.

[17] Harris, Charles R., et al. "Array Programming with NumPy." Nature, vol. 585 , no. 7825 , Sept. 2020 , pp. 357-62. www.nature.com, Available: https://doi.org/10.1038/s41586-020-2649-2.

[18] Stéfan van der Walt, Johannes L. Schönberger, Juan Nunez-Iglesias, François Boulogne, Joshua D. Warner, Neil Yager, Emmanuelle Gouillart, Tony $\mathrm{Yu}$ and the scikit-image contributors. scikit-image: 
Image processing in Python. PeerJ 2:e453 (2014) Available: https://doi.org/10.7717/peerj.453

[19] Chakrabarty, N., Apr. 2019. Brain MRI Images for Brain Tumor Detection. Available: https://www.kaggle.com/navoneel/brain-mriimages-for-brain-tumor-detection

[20] Mikołajczyk, A., dan Grochowski, M., 2018. "Data augmentation for improving deep learning in image classification problem". 2018 International Interdisciplinary PhD Workshop (IIPhDW). 117-122.
[21] Codeacademy. Normalization.

Available: URL:https://www.codecademy.com/articles/no rmalization

[22] Hanlon, J., 2016. Why is so Much Memory Needed for Deep Neural Networks? Available: https:/www.graphcore.ai/posts/why-is-so-muchmemor y-needed-for-deep-neural-networks

[23] Powers, David, dan Ailab. 2011. "Evaluation: From precision, recall, and F-measure to ROC, informedness, markedness \& correlation". Journal of Machine Learning Technologies. 